Questions vives

\section{Questions Vives}

Recherches en éducation

$\mathbf{N}^{\circ} 33$ | 2020

Visées heuristiques, praxéologiques et critiques dans les recherches participatives en éducation

\title{
Fonctions de la recherche et participation : une épistémo-compatibilité dans le cas de la recherche- intervention
}

Functions of research and participation: an epistemo-compatibility in the case of intervention research

Jean-François Marcel

\section{(2) OpenEdition}

Journals

Édition électronique

URL : http://journals.openedition.org/questionsvives/4691

DOI : 10.4000/questionsvives.4691

ISSN : 1775-433X

Éditeur

Université Aix-Marseille (AMU)

Édition imprimée

Date de publication : 15 décembre 2020

ISBN : 978-2-912643-57-5

ISSN : 1635-4079

\section{Référence électronique}

Jean-François Marcel, «Fonctions de la recherche et participation : une épistémo-compatibilité dans le cas de la recherche-intervention », Questions Vives [En ligne], № 33 | 2020, mis en ligne le, consulté le 25 mars 2021. URL : http://journals.openedition.org/questionsvives/4691 ; DOI : https://doi.org/ 10.4000/questionsvives.4691

Ce document a été généré automatiquement le 25 mars 2021.

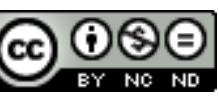

Questions Vives est mis à disposition selon les termes de la licence Creative Commons Attribution -

Pas d'Utilisation Commerciale - Pas de Modification 4.0 International. 


\title{
Fonctions de la recherche et participation : une épistémo- compatibilité dans le cas de la recherche-intervention
}

\author{
Functions of research and participation: an epistemo-compatibility in the case of \\ intervention research
}

Jean-François Marcel

1 La recherche en sciences de l'éducation et de la formation revendique traditionnellement, comme l'ensemble des recherches en SHS, la visée de production de savoirs scientifiques (ce qui renvoie à sa fonction heuristique) et une réflexion distanciée sur les conditions de cette production et sur sa diffusion et les modalités de son utilisation (ce qui renvoie à sa fonction critique). Or, ces deux fonctions se trouvent chahutées par la mobilisation potentielle d'une troisième, portant sur l'action et la pratique et ciblant leur efficacité, la fonction praxéologique. La perturbation est même renforcée et relayée lorsque le choix est fait de mobiliser une démarche participative, soucieuse de l'engagement de l'ensemble des acteurs au sein de cette démarche.

2 Cet article se propose d'instruire les conséquences du rapprochement des fonctions heuristiques et critiques avec la fonction praxéologique et ce, dans le cas où le choix d'une démarche participative génère un second niveau de perturbations. Il analyse les conditions de la mobilisation des trois fonctions en contexte épistémologique de participation, ce que nous avons qualifié, en titre, « d'épistémo-compatibilité ».

3 Pour ce faire, notre argumentaire s'appuie sur le cas de la démarche de rechercheintervention (désormais RI). Cette démarche est basée sur l'articulation des visées heuristiques, critiques et praxéologiques qui s'avèrent indissociables. Après l'avoir longtemps considérée comme un principe, de par sa transversalité, c'est plutôt dans la visée critique que nous situons l'émancipation de l'ensemble des participants, comme nous le développerons plus avant. Ces trois visées sont ancrées dans le principe premier 
et fondateur de la RI, celui de la participation. Si nous présentons la RI assez rapidement ici, nous développerons plus précisément sa composante participation, au centre de cet $\operatorname{article}^{1}$. Dans cette même logique participative, l'évaluation de la RI est aussi présentée.

Ensuite, pour mieux cerner l'épistémo-compatibilité de la participation avec chacune des trois fonctions de la recherche en éducation (auxquelles sont consacrées cette livraison de la revue), nous mobiliserons une analyse en deux étapes. La première aborde la participation à partir des trois fonctions tandis qu'à l'inverse la seconde investit les visées à partir des trois cercles qui correspondent aux niveaux de participation à l'œuvre dans la RI.

5 Précisons que, tout au long de ce texte, nous avons distingué les termes « fonction » et « visée ». Nous avons réservé «fonction» (au sens de fonction sociale et scientifique) à la recherche en éducation et « visées » (au sens d'objectifs, de finalités) à la RI, telle que nous l'avons théorisée.

\section{La participation structurante de la RI}

6 Le principe fondateur de la RI est celui de la participation (le « avec ») qui mobilise potentiellement tous les acteurs durant toutes les phases de la démarche et qui défend sinon une forme d'horizontalité tout au moins une absence de hiérarchie implicite. En lien avec la conduite et l'accompagnement du changement dans des contextes d'éducation, d'enseignement, de formation, de travail social, de soins, etc. et, à partir de sa base du « avec », la RI poursuit simultanément et en articulation trois visées ${ }^{2}$ qui correspondent aux trois fonctions de la recherche en éducation :

7 - une visée heuristique (le « sur ») qui mobilise un procès de recherche contextualisée en vue d'élaboration de savoirs scientifiques,

8 - une visée praxéologique (le " pour ») qui s'appuie sur les connaissances de acteurs sur leur action et sur les dynamiques collectives pour stabiliser des savoirs pour l'action et les mobiliser pour concevoir et mettre en œuvre des modalités de transformation

9 - une visée critique (le " par »). Au-delà d'une vigilance sur l'ensemble de la démarche (en terme de cohérence, de responsabilité, d'arbitrages éthiques, etc.), cette troisième visée accorde une place prépondérante à l'émancipation. L'émancipation est ici entendue comme un déplacement entre la place initialement occupée par un citoyen dans le champ professionnel et/ou social vers une place plus conforme à ses aspirations (Marcel, 2017). Cette dynamique est ici alimentée par l'hétérogénéité du collectif (statuts, visées, compétences, attentes, représentations, etc.) mobilisé au service d'un projet partagé et permettant de s'émanciper « ensemble ».

\subsection{Le choix de la participation}

Définissons brièvement ce que nous entendons par "participation » en convoquant d'abord Broudoux (2014) qui établit une gradation entre différents modes de contribution dans la communication scientifique. Pour lui, la participation serait la condition minimale: alors que collaborer supposerait une responsabilité plus importante dans la gestion et l'accomplissement des tâches, participer consisterait à simplement prendre part à une initiative en acceptant les règles proposées et en se 
conformant à des attendus. Sur la base de l'étymologie, nous contesterons cette hiérarchisation : "participer ", du latin participare, signifie "prendre part à " tandis que "collaborer" provient du latin laborare (travailler) et concerne donc la sphère laborale, uniquement le "travailler ensemble». Il s'avère donc plus restrictif que participer, de par sa référence explicite au travail. A l'inverse, la participation mobilise un empan plus large avec les modalités possibles de « prendre part à ». Ici l'engagement des participants apparait plus important, comme l'indique Ben Rajeb (2011) à propos du processus de conception architecturale :

"La démarche participative consiste à convier, les futurs usagers comme consultants ou conseillers à propos de leur espace à vivre. (...) Cette démarche aurait pour objectif de permettre aux maitres d'ouvrages et aux futurs utilisateurs concernés d'élaborer des décisions et des compromis collectifs concernant la définition du programme et de leurs espaces de vie ».

11 Le choix de la participation permet à la RI, d'une part, de potentiellement déborder du strict cadre laboral (et nous verrons, en suivant, que c'est le cas, notamment avec le $3^{\text {ème }}$ cercle) mais surtout d'assumer pleinement l'horizontalité du « avec » en enrôlant l'ensemble des acteurs jusqu'aux sphères de décisions et de négociations. En fait, la participation peut être à l'œuvre à tous les stades et pour toutes les tâches de la RI.

\subsection{La traduction du choix participatif : le TESS}

12 Le tiers-espace socio scientifique (TESS, Marcel 2010, 2020) assume, porte, garantit, opérationnalise, concrétise et fait vivre la RI. Cet espace correspond, en fait, au trait d'union de recherche-intervention et s'il est qualifié de socio-scientifique, c'est à deux niveaux interdépendants, celui des liens entre science et société que la RI met «au travail» et celui des contextes, professionnels et de recherche, que la RI relie, au travers d'une dimension participative, en articulant les trois visées déjà indiquées. Rappelons que la RI, en prise directe avec les demandes et les problématiques professionnelles ou sociales assume sa mission première d'accompagnement $\mathrm{du}$ changement (et, à un niveau moindre, de conduite du changement).

\subsubsection{La propédeutique du TESS : un « avec » potentiel}

13 Le TESS est institué par la signature de la commande qui en pose le cadre. Sur la base de ce cadre, il est co-élaboré selon trois cercles qui correspondent à la fois à trois étapes dans le déroulement de la RI et à trois modalités de participation se déployant au sein de contextes de plus en plus larges et ouverts. Cette co-élaboration mobilise la dialectique contrainte (du cadre) / liberté (des initiatives des acteurs) qui caractérise tout dispositif (Peeters \& Charlier, 1999). En revanche, il serait sans doute réducteur de faire démarrer la RI avec son officialisation et nous avons qualifié cette phase de germination de la RI de «propédeutique » pour affirmer la centralité du TESS.

La commande ne peut s'envisager que comme un premier résultat de la RI, le résultat d'une phase périlleuse qui pourrait sans doute se caractériser à l'aide de la métaphore de la procréation. Même s'il peut être suscité, provoqué, arrangé ou imprévu, nous commencerons par le premier rendez-vous, celui de la rencontre entre des représentants d'une Unité de Recherche (désormais UR) et ceux d'une "entreprise » (association, collectivité territoriale, organisme, etc.) porteuse d'une demande (souvent assez vague) de changement. En cas d'issue favorable, d'autres rendez-vous sont 
programmés. Ils ouvrent l'espace à des négociations (portant sur des volets divers : les attendus, les contraintes, les ressources, les finances, les temporalités, la faisabilité, etc.) qui deviennent de plus en plus précises, de plus en plus approfondies jusqu'à la construction d'un accord pour un engagement dans un projet partagé de RI. La formalisation, au sein du contrat que constitue la commande, suscite généralement quelques ajustements (ici aussi, cela "marche» rarement du premier coup) avant d'aboutir aux signatures. Dès lors, nous pouvons dire que la RI est née.

\subsubsection{Le premier cercle: une dynamique intrinsèque}

Le TESS prend vie avec l'installation des deux instances qui composent son premier cercle, le Comité de Pilotage (désormais CoPil) et le Groupe de Travail (désormais GTra), installation explicitement pilotée par la commande :

a. Le CoPil est composé des commanditaires (qui gouvernent «l'entreprise» concernée et financent la RI), des chercheurs et de deux représentants du GTra (qui le rejoindront dès que ce groupe aura démarré). Parfois un ou deux des partenaires de l'entreprise (locaux, régionaux, voire nationaux) s'y voient intégrés pour l'extériorité et la légitimation qu'ils fournissent. Le CoPil se réunit une à deux fois par an. Il a la charge de réguler le déroulement de la RI en terme de respect du calendrier ou des attendus : il assure ainsi une certaine plasticité à la commande. Ces régulations sont en lien direct avec les questions de faisabilité auxquelles est confrontée régulièrement le GTra et la présence de deux de ses représentants dans le CoPil enrôle véritablement ce dernier dans une démarche participative. Le CoPil ne «surveille » pas, il institutionnalise les avancées du GTra car il a aussi la charge de valider, au nom de l'institution qu'il représente, les propositions du groupe.

b. Le GTra est l'instance qui, concrètement, a la charge de la mise en œuvre du projet. Sur la base de la commande, reformulée et réappropriée, il organise son travail en fonction des visées. Il est composé de membres différents, des chercheurs et des acteurs de «l'entreprise» (occupant souvent des fonctions diversifiées) qui, au départ, ne se connaissent pas, mais qui sont réunis autour du projet posé par la commande. Ce groupe se constitue dans et par la réappropriation collective de la commande et se réunit régulièrement durant la réalisation du projet. Deux principes fondent le GTra :

- l'horizontalité et l'absence de hiérarchie explicite : chaque membre contribue à l'ensemble des étapes du projet, en fonction de ses compétences, de son expérience et de ses possibilités. La répartition des tâches se fait sur cette base et non sur leur valeur symbolique,

- la participation des professionnels : leur engagement se fait sur la base du volontariat et, surtout, la participation est incluse dans le temps de travail, sans empiéter sur le temps personnel. En revanche, l'engagement est associé à une responsabilité, en termes d'assiduité et de réalisation du projet annoncé.

Nous voyons que ce premier cercle de participation concerne directement un nombre réduit de participants (le CoPil et le GTra), un effectif circonscrit par la commande (ce qui nous autorise à qualifier la participation de "à l'interne"). Il se déploie dès le départ et aboutit à la stabilisation des « livrables », les ressources pour le changement, prévus par la commande. Il permet également la stabilisation de savoirs contextualisés (sur et pour l'action) co-élaborés par l'articulation de démarches heuristiques et de démarches praxéologiques. C'est aussi dans ce premier cercle que, de manière importante, se génèrent collectivement les processus d'émancipation professionnelle. 


\subsubsection{Le deuxième cercle : une dialectique intrinsèque / extrinsèque}

17 Avec le deuxième cercle s'amorce une socialisation "à l'externe » de la RI. Il s'agit d'une étape de valorisation de la RI qui s'appuie sur les livrables produits. Ces ressources pour le changement font alors l'objet de présentations, principalement au sein de «l'entreprise» mais aussi au sein de ses réseaux (locaux, régionaux, voire nationaux) selon des modalités diverses (journées d'études, capsules vidéos, exposition, articles professionnels).

Ce deuxième cercle permet de faire connaître et de faire reconnaître la RI, une dynamique impulsée collectivement par les mêmes acteurs, ceux du GTra renforcés selon le cas par ceux du CoPil. C'est aussi le cercle où se sont reconnus les avancées en termes de développement professionnels, à la fois les savoirs relatifs à faire son métier que ceux relatifs à être à son métier. Enfin, le $2^{\text {ème }}$ cercle a une fonction préparatoire pour le $3^{\text {ème }}$ en initiant la montée en généralité des savoirs contextualisés et la montée en puissance des ressources pour le changement.

\subsubsection{Le troisième cercle : une autonomie relative}

19 Même s'il est en liens étroits avec les deux précédents, le troisième cercle de participation s'en différencie en s'ouvrant à l'autonomie relative des deux partenaires (UR et "entreprise»), ce qui se traduit par une forme de dissociation, toutefois circonscrite par la consistance d'un collectif structuré et renforcé par l'expérience partagée des étapes précédentes. Il s'agit là d'une forme différente de participation et nous la retrouvons dans les deux contextes :

- au sein de l'UR, le travail scientifique de montée en généralité de savoirs contextualisés vers la stabilisation de savoirs scientifiques alimente une dynamique de publication et de diffusion à destination de la communauté scientifique. Précisons qu'il n'est pas exclu que des professionnels soient associés, selon des modalités à préciser, à ces publications.

- au sein de l'entreprise, les ressources pour le changement, dorénavant reconnu, font l'objet d'une forme d'industrialisation, c'est à dire d'une configuration adaptée à leur implémentation sur l'ensemble de "l'entreprise ». Ce processus est plutôt piloté par les professionnels et leur hiérarchie mais il n'est pas exclu que des chercheurs soient associés, selon des modalités à préciser, à cette implémentation.

20 C'est aussi dans ce troisième cercle que, de manière importante, se déploient individuellement les processus d'émancipation personnelle, que se réalisent les déplacements.

\subsection{L'évaluation de la RI}

21 Il est nécessaire de dire quelques mots complémentaires à propos du processus d'évaluation de la RI, d'une part parce qu'il s'agit d'un processus continu et longitudinal ${ }^{3}$ qui traverse l'ensemble des cercles du TESS et, d'autre part, parce qu'il est structuré , lui aussi, par le principe de participation. 


\subsubsection{Mobilisation de trois modèles de l'évaluation}

Évaluer la R-I, ses trois visées et son principe participatif, nécessite d'articuler plusieurs approches. Trois d'entre elles sont plus particulièrement mobilisées et nous les présentons brièvement (Vial, 1992 ; Marcel et Bedin, 2018) :

a. le modèle de la mesure : proche du " contrôle ", il convoque un référent « externe » à la RI et cible les résultats (changements en termes de savoirs, de transformations, contributions critiques) au travers d'approches quantitatives ;

b. le modèle des valeurs : il est basée sur le primat du sens et du vécu des acteurs, investit donc les processus de leurs élaborations (individuelles et collectives) en privilégiant des approches qualitatives ;

c. le modèle de la gestion : il interroge les processus de transformations générées par la RI, tant au niveau individuel et collectif des acteurs (développement professionnel, émancipation, etc.) qu'au niveau organisationnel (régulations, pilotage, aide à la décision, accompagnement de l'appropriation des transformations, etc.) en mobilisant prioritairement des approches qualitatives.

\subsection{2. Évaluation et participation}

La participation est certes à l'œuvre selon des formes différenciées en fonction des trois modèles mais nous autorise à parler de co-évaluation. Cette dernière est d'ailleurs repérable dans la phase propédeutique du TESS durant laquelle les avancées des négociations s'apparentent à une démarche de référentialisation et la formalisation de la commande à la stabilisation de la référence de la RI. La co-évaluation se déploiera d'ailleurs principalement au sein de l'empan entre la commande (le référent instituant «l'attendu») et les changements co-produits (scientifiques, socioprofessionnels, politiques constituant «l'obtenu»). Les modalités de cette co-évaluation dévoilent la prééminence d'un modèle d'évaluation dans chacun des cercles du TESS, même si bien sûr, il convient de les envisager comme poreux, complémentaires et interdépendants.

Ainsi, dans le cercle 1, le processus de référentialisation, initié dans la propédeutique, se prolonge au travers de l'appropriation et de la mise en œuvre du projet partagé. Cette élaboration du "en commun" est propice à la mise en dialogue (ou en confrontation) des valeurs individuelles et du sens des engagements, jusqu'à la stabilisation d'un socle axiologique partagé et d'une visée herméneutique commune. Le cercle 1 sera donc préférentiellement investi par le modèle des valeurs.

Le cercle 2 est plutôt dominé par les processus de reconnaissance. En suivant Ricœur (2004), Lazzeri \& Caillé distinguent deux aspects (cognitif et pratique) de ce processus. Pour l'aspect cognitif, la «compétence d'identification» correspond à «ce que l'on connaît ou dont on anticipe la nature sur un mode non assuré, (et qui) se trouve confirmé par une opération de subsomption par laquelle on place la chose à connaître sous la juridiction d'un concept » (Lazzeri et Caillé, 2004, p. 89). L'aspect pratique de la reconnaissance se caractérise, lui aussi,

«par un acte d'identification qui revêt la forme d'une attestation », « une demande de confirmation de la valeur des actions et des capacités de la part d'un individu (ou d'un groupe) lorsqu'il éprouve un doute à ce sujet et qu'il s'adresse à son environnement social afin d'obtenir cette confirmation " (Lazzeri \& Caillé, 2004, p. 90).

Nous repérons là deux dimensions qui, au sein de la RI, se retrouvent dans la phase de valorisation. En effet si l'aspect pratique est évident, l'aspect cognitif apparaît comme 
indissociable : toute forme d'apprentissage ou de développement requiert une forme de reconnaissance sociale pour être stabilisé et validé. Le cercle 2 sera donc préférentiellement investi par le modèle de la gestion, celui des transformations des acteurs et de l'organisation (transformations reconnues au sein du contexte socioprofessionnel).

Le cercle 3 est celui d'une autonomie relative (puissamment ancrée dans ce «en commun » déjà rappelé), une autonomie des chercheurs et des professionnels au sein de leurs contextes d'origines, avec le processus de diffusion des publications au sein du contexte académique et avec le processus d'implémentation des transformations au sein du contexte professionnel (sans exclure des modalités complémentaires comme, par exemple, des publications dans des revues professionnelles relevant de réseaux de diffusion locaux ou nationaux). Dans les deux cas, l'efficacité en termes de résultats va s'ériger en boussole, ce qui correspond, en exacerbant la logique, à l'impact-factor d'un côté et au rendement de l'autre. Bien sûr, la visée politique permettra de réguler ces dérives mais nous pouvons dire que le cercle 3 sera préférentiellement investi par le modèle de la mesure.

\section{La participation dans la RI via les trois fonctions de la recherche}

28 La première étape de l'analyse investit la participation dans la RI (à laquelle a été consacré le paragraphe précédent) à partir des trois fonctions et permet de mieux cerner comment les visées de la RI «contextualisent » les fonctions de la recherche en éducation.

\subsection{La fonction heuristique et les validités scientifiques}

La fonction heuristique correspond à la production de savoirs scientifiques. Elle constitue, tant historiquement, socialement que scientifiquement, l'étendard de la recherche. Elle se caractérise par sa stricte structuration autour d'une problématisation (potentiellement déclinée en hypothèses), par le respect rigoureux de méthodes, par la mobilisation explicite de cadres théoriques, par une confrontation à l'empirie, par des principes (transparence, reproductibilité, falsification, validités), le tout encadré par des règles déontologiques fortes (voire des arbitrages éthiques). Elle vise à la production de savoirs au service du progrès de l'humanité et, en ce sens, tendant vers une forme d'universalité (parfois dénoncée comme une vanité par la métaphore de la tour de Babel).

\subsubsection{Les profanes et le contexte}

Or, au sein de la RI, la visée heuristique présente deux spécificités. La première, en lien avec son principe participatif, est de revendiquer la légitimité et la pertinence de la contribution, au procès de recherche, de professionnels qui ne sont pas formés à la recherche $e^{4}$ et de défendre non seulement qu'elle est possible (ce procès ne s'en trouvera pas perverti) mais qu'elle est souhaitable (il en ressortira enrichi).

31 La seconde spécificité est que la RI se doit de gérer une tension fondatrice entre, d'une part, une dynamique de contextualisation (de la problématique, de l'enquête et des 
résultats, en lien avec la demande initiale) et d'autre part, comme dans toute recherche, une dynamique de généralisation (relevant de la validité scientifique externe) qui préserve les exigences de cohérence et de consistance du procès de recherche (relevant de la validité scientifique interne).

\subsubsection{Trois niveaux de validité scientifique}

Pour être plus précis, nous déclinerons la validité scientifique selon trois niveaux. En ce qui concerne la validité interne, c'est-à-dire la cohérence du procès de recherche et la consistance de l'articulation entre les différentes composantes de ce procès, elle demande certes une attention non négligeable mais assez habituelle, surtout que, dans la plupart des cas, les RI mobilise des démarches qualitatives.

La validité externe est tout à fait spécifique puisqu'elle porte sur la généralisation des avancées. Nous distinguerons deux niveaux. Le premier, à partir des connaissances des professionnels et des méthodologies de recherches, concerne la co-élaboration de savoirs contextualisés à l'organisation au sein de laquelle est déployée la RI (et qui est strictement inscrite dans la commande). Cette première étape de la montée en généralité s'effectue de manière fortement participative et ces savoirs contextualisés sont dotés, en plus d'une solide validité interne, d'une certaine validité scientifique externe mais, surtout, en même temps sont potentiellement utiles aux professionnels (et donc se voient dotés d'une certaine validité sociale) ${ }^{5}$.

Le second niveau de validité externe peut, dans le cas des recherches qualitatives ${ }^{6}$, emprunter deux voies différentes pour assumer une montée en généralité plus importante, largement émancipée du contexte de production et tendant vers l'universalité. La première voie est la mise en dialogue des savoirs contextualisés coconstruits avec des savoirs scientifiques disponibles. Ce dialogue adopte des modalités diversifiées (complément, précision, contradiction, alternative, etc.) et positionne ces savoirs au sein du $3^{\text {ème }}$ monde ${ }^{7}$ défini par Popper (1991), celui des idées. La seconde voie de généralisation mobilise une comparaison inter RI, une mise en relations de savoirs contextualisés co-élaborés dans des RI différentes. Cette mise en relations dégage des spécificités mais également des invariances sur la base desquelles peut être conceptualisée une importante montée en généralité.

\subsection{La fonction praxéologique et la validité sociale}

La fonction praxéologique repose sur un primat de l'action ${ }^{8}$, sur un travail de praxis ${ }^{9}$, sur "la destruction des barrières qui ont divisé la théorie et le pratique " (selon la formulation de Dewey, 1929) et sur la primauté de l'acteur ${ }^{10}$.

\subsubsection{Les trois principes}

Mobilisons la définition de Lhotellier et St-Arnaud (1994) : "La praxéologie est une démarche construite (visée, méthode, processus) d'autonomisation et de conscientisation de l'agir (à tous les niveaux d'interaction sociale) dans son histoire, dans ses pratiques quotidiennes, dans ses processus de changement et dans ses 
conséquences » (p. 95). Ils distinguent trois principes méthodologiques, la connaissance par l'action, la coopération dialogique et l'autorégulation :

- Le principe de la connaissance par l'action défend l'autonomisation d'un savoir ${ }^{11}$ et la possibilité d'une « interaction entre le savoir et l'action (...) pour la progression de l'agir vers une plus grande efficacité $\aleph^{12}$ (Lhotellier et St-Arnaud, 1994, p. 96).

- Le principe de la coopération dialogique rappelle que le savoir se construit d'abord par un dialogue entre l'acteur et la situation et « la praxéologie vise à articuler ce dialogue, à lui donner une rigueur qui permettra la diffusion et l'enrichissement éventuel du savoir homologué » (Lhotellier et St-Arnaud, 1994, p. 101). La construction du savoir a comme corollaire celle du sens, «le sens n'existe pas en soi, il est un produit, une création », mais cette construction requiert une ouverture aux autres, des modalités de collaboration: «Le travail du sens qui est proposé est pluriel; il exige un partenariat: c'est en variant les discours que l'on peut créer du sens nouveau » (Lhotellier et St-Arnaud, 1994, p. 101). Nous retrouvons ici un lien explicite avec l'approche participative, promue par la RI.

- Le principe de l'autorégulation correspond aux arbitrages de l'acteur, lui permettant de ne retenir que ce qui contribue à la progression de l'action. En revanche, ses arbitrages ne peuvent s'envisager qu'au sein d'un espace collectif qui légitimera le sens de l'action: "Sachant qu'une action ne peut être isolée de son contexte culturel et historique, la pluralité des regards et des discours, donc la confrontation, devient le moyen privilégié pour valider et déployer le sens d'une action » (Lhotellier et St-Arnaud, 1994, p. 102). Il semble dès lors que le préfixe « auto » ne se justifie plus vraiment puisque la dimension collective est affirmée : nous parlerons donc du "principe de régulations ».

En résumé, les trois principes de la praxéologie mettent au jour un processus dialectique entre individuel et collectif, en large adéquation avec une démarche participative : «En vertu du principe de la connaissance par l'action, l'acteur est le premier interprète de son action; en vertu du principe de coopération dialogique, l'acteur a besoin d'être exposé à toutes sortes de discours pour dégager tout le sens de son agir » (Lhotellier et St-Arnaud, 1994, p. 102).

\subsubsection{L'efficacité}

Nous avons pu constater que la participation était non seulement épistémo-compatible avec la fonction praxéologique mais qu'elle lui était également nécessaire. En revanche, deux points méritent d'être précisés.

Le premier concerne l'action. Nous avons abordé les savoirs qu'elle permet d'élaborer, son sens qui requiert d'être co-construit, la quête de son efficacité. Or, dans le cas de la RI, il conviendrait de rajouter deux dimensions, d'une part son inscription au sein d'un projet partagé (qui renforce le volet collectif) et il s'agirait, d'autre part, de s'attarder sur le résultat de cette action, en lien avec le changement qui sous-tend la commande. Ainsi, l'efficacité de l'action se doit d'intégrer un triple niveau d'efficacité : efficacité pour l'acteur, efficacité pour les partenaires directs du groupe de travail et efficacité pour les commanditaires de la RI (explicitée par la commande). Les résultats de l'action renvoient, certes, aux transformations produites par la RI mais la visée praxéologique revendiquée permet de bien la distinguer d'une simple visée transformative qui se trouverait cantonnée dans l'activisme et l'empirisme. niveaux d'efficacité «à l'interne » de la RI, il convient d'envisager une efficacité «à 
l'externe », d'une part au travers des formes de valorisation (une efficacité en termes de reconnaissance sociale) et, d'autre part, en terme d'implémentation de transformations au sein de l'organisation (une efficacité en termes de changement). Cette efficacité «à l'externe » correspond à la validité sociale de la RI qui se construit sur la base de la réception, de l'appropriation et de l'implémentation des transformations, des ressources professionnelles co-élaborées. En relation avec la commande, elle se base sur le changement effectif au niveau de l'organisation et s'émancipe assez largement du contexte de son élaboration. Elle est placée sous le primat de l'appréciation, par des professionnels, d'une plus-value pour l'organisation, d'une amélioration de leur travail.

\subsection{La fonction critique}

41 Nous défendons qu'une dimension critique demande à être préservée au sein de nos recherches mais qu'elle nécessite, sans doute, d'être promue et revendiquée : cette section en esquisse les modalités.

\subsubsection{Les trois dimensions de la critique}

Lévy-Leblond (1996) caractérise trois dimensions de l'activité critique que nous prolongerons, chaque fois, à l'aide des propositions de De Munck (2011) qui s'avèrent en forte adéquation avec elles :

- La première dimension, qualifiée de productrice, cible le processus de recherche «à l'interne ». Elle concerne la validité du travail scientifique (une vigilance par rapport à la validité interne) mais aussi sa qualité (au travers d'un souci d'exigence). L'auteur y rajoute la signification ou le sens du travail (notamment la prise de distance à l'aide d'un temps suffisamment long pour permettre de saisir, de comprendre l'ensemble de la démarche, sa structure) mais aussi son orientation (en repérant et explicitant l'éventail des choix possibles pour être en mesure d'évaluer les enjeux de ceux retenus). Enfin, il insiste sur l'importance de l'historicité en positionnant cette recherche certes par rapport aux traditions historiques mais surtout par rapport au contexte scientifico-historique actuel. Nous pourrions la prolonger avec la critique (interne) dont De Munck (2011) relève l'inspiration kantienne, celle d'une science non dogmatique et qui fait de la raison l'arme privilégiée de lutte contre les préjugés irrationnels. Il apparaitt que cette dimension critique productrice concerne principalement la visée heuristique.

- La deuxième dimension de l'activité critique (Lévy-Leblond, 1996), qualifiée de médiatrice, concerne les processus de diffusion, «à l'externe " donc. Dans ce cas, il ne s'agit pas seulement de savoir ce qui est vrai, mais de savoir en quoi ce vrai est ou peut être important ou intéressant. Dans cette même logique, d'inspiration herméneutique et pragmatique, De Munck (2011) invite à ne pas faire l'impasse sur les jugements de valeur, à ne pas prétendre occulter le système normatif secrété par l'explication du monde du chercheur. En fait, il défend l'intérêt d'une mise en relation du cognitif et du normatif par le langage évaluatif. Il apparaît que cette dimension critique médiatrice concerne principalement la visée praxéologique.

- La troisième dimension de l'activité critique (Lévy-Leblond, 1996), qualifiée de politique, concerne les processus de socialisation de la recherche, processus que nous avons détaillé dans les deuxièmes et troisièmes cercles du TESS. De Munck (2011) repère son inspiration marxiste et la précise en invitant le chercheur à mobiliser sa réflexion sur les possibilités et 
les limites de son propre exercice, notamment dans ses stratégies pour construire des liens, une convergence entre ses pratiques, qu'elles soient professionnelles, sociales ou militantes, et les orientations et les avancées de ses travaux scientifiques, étant donné qu'ici, la dimension critique mobilise la recherche en vue de transformer le monde. Il apparait que cette dimension critique politique d'une part s'alimente aux avancées des visées heuristiques et praxéologiques mais, surtout, qu'elle s'efforce de les articuler pour ensuite dégager une marge d'autonomie. Cette marge d'autonomie (dont la maîtrise apparaît comme un réel enjeu, notamment en terme de crédibilité dans le débat social) constitue une sorte de levier, voire de tremplin, pour participer et contribuer à un changement du monde, voire à un changement de monde.

\subsubsection{Visée critique de la RI et émancipation}

Ces trois dimensions renforcent la cohérence et la consistance de la RI. En effet, sous l'égide et la dynamique d'une démarche participative, la mise en lien des fonctions productrice (concernant plutôt la visée heuristique) et médiatrice (concernant plutôt la visée praxéologique) avec la dimension politique, éclaire différemment le processus d'émancipation ${ }^{13}$.

Nous définissons l'émancipation (Marcel, 2017) d'abord par la force d'un collectif ${ }^{14}$ qui en constitue le potentiel énergétique et la dynamique principale. Il se caractérise par l'hétérogénéité des statuts, des spécialités et des membres (chercheurs, professionnels) et se structure par et autour d'un projet commun ${ }^{15}$. Toutefois, ce collectif est surtout dépendant de l'abolition de toute hiérarchie symbolique $^{16}$.

Bien sûr, l'émancipation assume une portée peut être subversive, sans doute iconoclaste au travers de la reconfiguration des espaces, incluant le franchissement de frontières. En effet, la RI entend permettre, grâce aux dynamiques collectives, le « déplacement » de chacun des acteurs. Ce déplacement consiste simultanément, pour chacun d'eux, à s'extraire du positionnement auquel il ou elle se trouvait assigné au départ et à conquérir un positionnement plus conforme à ses aspirations. Ce positionnement peut concerner, pour tout ou partie, des dimensions professionnelles (comme l'accès à de nouvelles fonctions), des dimensions sociales (comme le choix de nouveaux engagements, politiques ou militants), des dimensions individuelles (comme le développement du pouvoir d'agir), des dimensions symboliques (comme l'accès à de nouvelles formes de reconnaissance). Enfin, l'émancipation mobilise une dimension herméneutique qui mobilise un processus individuel de réappropriation de ses temps. En effet, si l'émancipation repose ici sur un engagement intense au sein du collectif, celui-ci n'est déployé que sur une période courte et limitée, celle de la durée du projet. Ce constat, outre qu'il invite à une modestie certaine, est sans doute celui qui caractérise le plus fortement cette nouvelle approche de l'émancipation, coupée de grands collectifs stables et permanents, à l'image des grands récits dans lesquels ils s'inscrivaient jusque là. Ici, l'émancipation repose sur l'individu, véritable héros existentialiste proche de Sisyphe (Camus, 1985), condamné à reconstruire et à inventer perpétuellement une dynamique et une cohérence entre des collectifs et des engagements transitoires et temporellement bornés.

Il convient de rajouter deux précisions :

a. nous appelons émancipation professionnelle, le processus d'émancipation qui se déploie préférentiellement au sein du contexte professionnel. Pour appréhender cette catégorie de "déplacement ", nous l'avons décliné, dans nos enquêtes empiriques, à l'aide de quatre indicateurs : le sentiment d'efficacité professionnelle (se juger aujourd'hui capable de dire, de faire, d'intervenir, de penser, alors que ce n'était pas le cas avant), 
l'autorisation (aujourd'hui, vous vous autorisez à dire, à faire, à intervenir, à penser, alors que ce n'était pas le cas avant), le pouvoir d'agir (aujourd'hui, vous osez dire faire, intervenir, penser, alors que ce n'était pas le cas avant) et la légitimité (qui reconnaît votre légitimité pour dire, faire, intervenir, penser, alors que ce n'était pas le cas avant?).

b. l'émancipation mobilise nécessairement l'exercice de la responsabilité, à la fois versus chercheur (sa responsabilité par rapport à l'après-RI et à ses conséquences) et versus participants (en lien avec le changement qu'ils contribuent à impulser). Cet exercice est indissociable, au-delà du balisage des règles déontologiques, du recours à des arbitrages éthiques, tant au niveau collectif qu'au niveau individuel. Dans les deux cas, les modalités délibératives peuvent être mobilisées.

\subsection{Participation et visées : synthèse}

Cette section a permis de mettre au jour les liens et les connexions entre la dimension participative de la RI, telle que déployée au travers des trois cercles du TESS, et les trois visées de la RI (en tant que contextualisations des fonctions de la recherche en éducation) :

- En ce qui concerne la visée heuristique, la place de la participation est un choix assumé et revendiqué par les fondements épistémologiques de la RI. Ces fondements, au travers de la théorisation d'une tierce voie, défendent une vision de la relation entre science et société qui s'alimente à une articulation entre recherche et action (Marcel, 2019a). Il s'agit là d'un engagement, en terme de politique scientifique, qui se traduit également dans une dynamique de participation basée sur l'absence de hiérarchie. Cette horizontalité essentielle n'exclut pas la reconnaissance des compétences spécifiques de chacun des membres et leur mobilisation explicite au service du projet partagé.

- Dans le cas de la visée praxéologique, nous l'avons vu, la participation est un passage obligé. Si le principe de la connaissance par l'action reconnaît l'acteur comme premier interprète de son action, il reste inséré dans des interactions sociales (au sein des instances et principalement du GTra) pour la légitimation de son efficacité et pour la co-production de son sens (principe de la coopération dialogique). La participation se retrouve égalent dans le principe des régulations, où les arbitrages, qui permettent d'éviter de dériver soit du côté d'une connaissance purement spéculative soit du côté d'une action strictement empirique, sont des arbitrages collectifs.

- Enfin, la participation apparaît comme constitutive de la visée critique de la recherche, à la fois : dans les dimensions productrices «à l'interne " qui ciblent la mise à distance des croyances irrationnelle, et pour lesquelles le concours du collectif est requis; dans les dimensions médiatrices «à l'externe» qui initient, collectivement, une processus de socialisation de la RI et dans les dimensions politiques, qui rappellent les responsabilités des participants à la RI, dans leurs engagements professionnels mais surtout sociaux.

Par rapport aux trois dimensions, nous constatons que nous avons donc une participation choisie (que revendique la visée heuristique de la RI), une participation nécessaire (qu'opérationnalise la visée praxéologique de la RI) et une participation fondatrice (qu'assume la visée critique de la RI). Dès ce premier niveau d'analyse, la participation apparaît donc épistémo-compatible avec les trois fonctions de la recherche en éducation. 


\section{Les trois fonctions de la recherche via la participation dans la RI}

La seconde étape de l'analyse, à l'inverse de la première, investit les visées à partir des trois cercles du TESS qui correspondent aux niveaux de participation à l'œuvre dans la RI. Elle permet de préciser comment, à chacun de leurs niveaux, la RI prend effectivement en charge les trois fonctions de la recherche, comment elle les contextualise à l'aide de ses trois visées, comment est à l'œuvre cette «épistémocompatibilité $»^{17}$ à laquelle est consacrée l'article.

\subsection{Une synthèse de l'épistémo-compatibilité}

Afin d'éviter les redites et les redondances avec les paragraphes précédents, nous synthétiserons les différents éléments en les reconfigurant au sein du tableau suivant :

Tableau 1 : Synthèse de l'épistémo-compatibilité

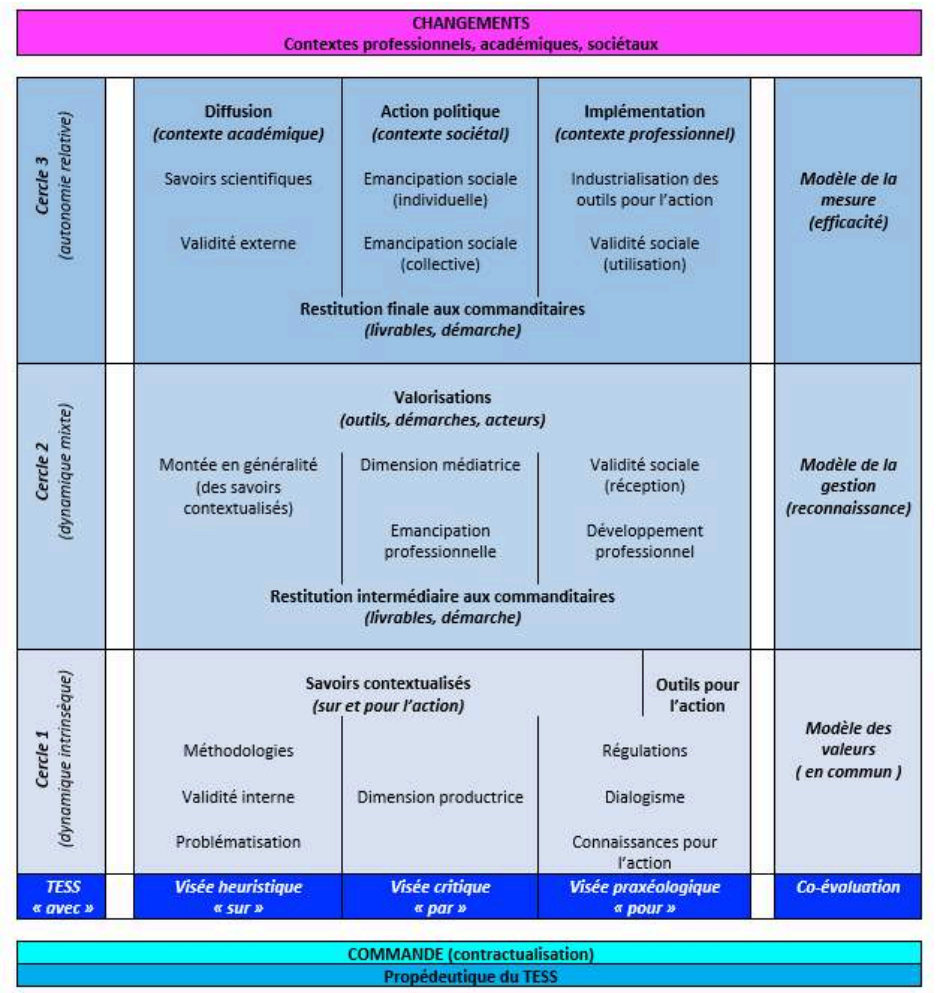

Nous développerons en suivant chacun des trois cercles en insistant sur l'interdépendance des trois visées de la RI et en mettant en évidence le rôle structurant d'une co-évaluation longitudinale (qui se pense, bien sûr, en lien avec la commande).

\subsection{Premier cercle : la construction du « en commun »}

Le cercle 1 de la participation, mis sur les rails par la commande, est fortement structuré par le projet, partagé après appropriation et polarisé de manière concrète par la réalisation du livrable (notamment les outils pour l'action). La visée praxéologique occupe une place importante puisque l'ensemble de la RI prend un premier appui sur 
les connaissances de et sur l'action (régies ensuite par le principe dialogique et par celui des régulations). Au sein de la RI, cette visée est en interrelation avec la visée heuristique (selon le pari de la tierce voie), visée heuristique qui convoque un procès de recherche étroitement connecté à la démarche praxéologique. La phase de problématisation est alors particulièrement importante en ce sens qu'elle permet de s'extraire, du moins en partie, de la situation et des pratiques de la visée praxéologique. Cette phase de problématisation est doublement étayée par la convocation des savoirs scientifiques concernant la thématique étudiée (une sorte d'état de la question) et par la mobilisation d'un cadre théorique (permettant d'instruire la thématique). Dans un second temps, les démarches méthodologiques (construction des outils, collectes empiriques, analyses) permettront de produire des résultats. Nous les avons qualifiés de « savoirs contextualisés » d'une part parce qu'ils sont formalisés (à la différence des connaissances tacites initiales), ils préservent un lien fort avec l'action (de laquelle la démarche praxéologique a accompagné l'extraction) et se dotent d'une validité scientifique. Cette validité est, certes, dotée d'une portée limitée en termes de validité externe, mais préserve une validité interne puissante, ciblant la cohérence et la consistance du procès de recherche. Elle permet en cela de prendre en charge la dimension productrice de la visée critique qui se voit adjoindre, ici, un volet complémentaire. En effet, il lui incombe d'exercer sa vigilance sur l'articulation des autres visées afin qu'elles évitent, à la fois, le Charybde de la dissociation et du cloisonnement et le Scylla de la confusion.

51 Dans ce cercle 1, la participation est également à l'œuvre avec la co-évaluation et l'importance de la co-élaboration du "en commun ", à la fois au travers des instances mais également du projet et de l'action partagés, rend ce cercle propice à être investi, préférentiellement, par le modèle des valeurs.

\subsection{Deuxième cercle : la conquête de la reconnaissance}

52 Si le cercle 1 a construit, plutôt à l'interne, à la fois des savoirs, des livrables et du commun, le cercle 2 va devoir effectuer une première ouverture "à l'externe ». Il va s'agir de donner à voir pour conquérir une reconnaissance que, seuls, les autres sont en mesure de lui fournir.

Il s'initie par la phase de restitution intermédiaire qui constitue le bilan du GTra (régulé par le CoPil). Les livrables et la démarche sont présentés lors d'un CoPil (sans doute un peu plus solennel) qui aura la charge de valider cette première phase. Il accordera ainsi un premier niveau de reconnaissance au GTra (pour son travail et pour le produit de son travail).

Il s'agira ensuite d'entamer la valorisation, de montrer la pertinence et l'intérêt du travail fourni en le confrontant à un public à dominante professionnel, certes restreint, mais allant au-delà de l'entre-soi. A ce stade, le CoPil est également enrôlé dans la valorisation d'une démarche et de livrables qu'il a validés. C'est l'opération qui permettra la conquête d'une reconnaissance solide, à la fois pour les livrables, pour la démarche et pour les acteurs (GTra et CoPil).

Au travers de la reconnaissance pour les livrables et pour la démarche, nous retrouvons la validité sociale, promue par la visée praxéologique. En ce qui concerne les acteurs, la dimension médiatrice de la visée critique va les conduire à élaborer des stratégies de valorisation pertinentes, en intégrant des contraintes externes, nouvelles dans cette RI. 
Cette stratégie va générer un développement de leur empan en termes d'autorisation et de pouvoir d'agir, et contribuer ainsi, fortement, à leur émancipation professionnelle. Ils initient ainsi leurs " déplacements », au sein de leur contexte professionnel. De plus, la reconnaissance conquise renforcera, bien sûr, ce processus émancipateur mais validera également leur développement professionnel. La RI n'est pas une démarche formatrice, mais le développement professionnel est toutefois logiquement intégré à la visée praxéologique, au service d'une action professionnelle toujours plus efficace, toujours plus réfléchie.

Dans le cercle 2, la visée heuristique n'est ni occultée ni escamotée, car, à la fois sa contribution à la stratégie de valorisation et l'analyse de la réception des outils, de la démarche, voire des savoirs contextualisés, par un public externe, vont lui être fort utiles pour affiner les savoirs contextualisés et alimenter leur montée en généralité.

Le cercle 2 est celui d'une première socialisation de la RI. Dès lors, la co-évaluation va cibler le processus de reconnaissance, ce qui rend ce cercle propice à être investi, préférentiellement, par le modèle de la gestion.

\subsection{Troisième cercle : l'essaimage}

Le cercle 3 de la participation s'ouvre par le CoPil de restitution finale, celui qui met un terme officiel à la démarche et aux instances. Pourtant la RI n'est pas vraiment terminée et la dynamique participative se poursuit. Certes, chacun des partenaires va continuer de manière plus autonome (ce qui n'exclut des modalités de collaborations sans doute plus ponctuelles) mais l'ancrage dans un « en commun » co-élaboré, qui plus est estampillé par cette reconnaissance collectivement conquise, va constituer une prévention à toute rupture radicale. De manière certes plus lâche, la participation se poursuit. Elle investit trois contextes, directement en lien avec chacune des visées :

- la visée heuristique cible le contexte académique au travers du processus de diffusion de publications. Ces dernières sont alimentées par les savoirs scientifiques, élaborés sur la base des savoirs contextualisés en respectant les exigences de validité externe, sous la responsabilité des chercheurs (sans exclure, potentiellement, la participation de professionnels),

- la visée praxéologique investit le contexte professionnel ${ }^{18}$, potentiellement à plusieurs échelles. Les outils pour l'action, validés et reconnus, sont maintenant en mesure d'être configurés pour être implémentés dans l'entreprise (nous avons parlé d'industrialisation pour caractériser cette montée en puissance). L'ensemble du processus est accompagné par les professionnels des instances de la RI (sans exclure, potentiellement, la participation de chercheurs),

- la visée critique s'exerce en contexte sociétal, au travers d'engagements (paroles, écrits, actions, etc.) des chercheurs et des professionnels, en lien plus ou moins direct avec le projet de RI. Bien sûr, au delà du thème lui même (qui requerra toutefois une vigilance importante pour ne pas le bafouer ou le renier), ce sont les différents niveaux du processus d'émancipation qui seront ici mobilisés : un niveau collectif, en lien avec la reconnaissance de la RI dans son ensemble et, surtout, un niveau individuel, en lien avec ce déplacement, cet accès à une place plus conforme aux aspirations des acteurs (chercheurs ou professionnels) qui caractérise la visée critique.

Le cercle 3 ouvre la RI sur de nouveaux contextes. Par conséquent, la co-évaluation va se trouver soumise aux règles de ces contextes et de leurs attendus, en termes 
d'efficacité. Dès lors, ce cercle demande à être investi, préférentiellement, par le modèle de la mesure. En revanche, la visée critique permet, nous l'avons dit, de circonscrire toute dérive potentielle vers l'idéal néolibéral d'un rendement à tout crin.

\section{En guise de conclusion}

60 À partir du cas de la RI, cet article a explicité les modalités selon lesquelles cette démarche de RI assumait, par ses visées, la mise en œuvre concrète des trois fonctions de la recherche. Pour ce faire elle a d'abord assumé un premier niveau « d'épistémo compatibilité » entre les visées heuristiques et praxéologiques, au travers de la théorisation des "savoirs contextualisés ", caractérisés par leur double validité, à la fois scientifique et sociale. Ils constituent une étape tant dans la démarche praxéologique (puisqu'ils sont mobilisés au service de l'efficacité de l'action et de l'élaboration d'outils pour l'action) que dans la démarche heuristique (puisque, au travers d'une montée en généralité respectant les exigences de validité externe, ils permettent de produire des savoirs scientifiques).

61 L'article a également montré l'interdépendance des trois visées de la RI, principalement de la visée critique qui, certes, est fortement liée aux deux autres qu'elle accompagne et qui l'alimentent, mais qui préserve toutefois une autonomie à deux niveaux corrélés, celui des processus d'émancipation et celui de la portée politique de la RI, en contexte sociétal.

Le texte a ensuite examiné comment le principe premier et fondateur de la RI (la participation) s'avérait, dans le cas de la RI, "épistémo-compatible » avec ses trois visées. Il a d'abord précisé la mise en œuvre de cette participation au travers de la coélaboration du TESS et de ses trois cercles de socialisation de la RI (dont on retrouve un écho dans les modalités de co-évaluation de la RI). Il a ensuite soumis l'articulation du TESS (la participation) avec les trois visées de la RI (contextualisation des fonctions de la recherche en éducation) à une analyse, selon deux étapes successives et complémentaires, privilégiant une entrée par les trois fonctions de la recherche pour la première et une entrée par la participation (et ses trois cercles) pour la seconde. Toutes deux ont réaffirmé "l'épistémo compatibilité » du principe fondateur (celui du « avec ») et des visées poursuivies par la RI (celles du « sur », du « pour » et du « par »).

Il ne serait sans doute pas inutile qu'un travail similaire d'évaluation de "l'épistémo compatibilité» soit effectué pour d'autres démarches s'identifiant comme participatives voire collaboratives ${ }^{19}$ d'autant que, comme nous l'avons développé par ailleurs (Marcel, 2020), nous défendons que ce grand courant des recherches « avec ", d'une part, constitue une perspective de développement pour les sciences de l'éducation et de la formation (en restant vigilant par rapport à quelques dérives potentielles que nous ne reprenons pas ici, voir Marcel, 2019c) et, d'autre part, s'inscrit dans un mouvement scientifique pluridisciplinaire, mobilisateur et très porteur, celui des « sciences participatives » (voir à ce propos, Robert \& Marcel, 2019). 


\section{BIBLIOGRAPHIE}

Ben Rajeb, S. (2011), Collaboration, coopération ou participation ?, DNArchi, 09/12/11, http:// dnarchi.fr/culturenumerique/collaboration-cooperation-ou-participation

Broudoux, E. (2014). Participer, coopérer et collaborer en milieu scientifique. Les cahiers de la SFSIC, 178-181.

Broussal, D. (2018). S'émanciper par les œuvres. Proposition pour la recherche-intervention. Paris : L'Harmattan.

Camus, A. (1985). Le mythe de Sisyphe. Paris : Gallimard.

De Munck, J. (2011) Les trois dimensions de la sociologie critique, Sociologies [En ligne], La recherche en actes, Régimes d'explication en sociologie. URL: http://journals.openedition.org/ sociologies/3576

Dewey, J. (1929). The Quest for Certainty. A Study of the Relation of Knowledge and Action, New York, Minton, Balch.

Gurnade, M-M. \& Marcel, J-F. (2016). La restitution comme espace de confrontation de savoirs pluriels : le cas d'une recherche-intervention. Nouveaux c@hiers de la recherche en éducation, 18 (2), 31-55. https://doi.org/10.7202/1036032ar

Lazzeri, C. \& Caillé, A. (2004). La reconnaissance aujourd'hui. Enjeux théoriques, éthiques et politiques du concept, Revue du MAUSS, 2004-1, n 23, p. 88-115.

Lévy-Leblond, J-M. (1996). La pierre de touche. La science à l'épreuve ... Paris : Gallimard.

Lhotellier, A. \& St-Arnaud, Y. (1994). Pour une démarche praxéologique. Nouvelles pratiques sociales, 7 (2), 93-109. https://doi.org/10.7202/301279ar

Marcel, J.-F. (2010). Des tensions entre le « sur » et le « pour » dans la recherche en éducation : question(s) de posture(s). Cahiers du CERFEE, 27-28, 41-64.

Marcel, J.-F. (2013). Contribution à une ingénierie de la commande, TransFormations-Recherches en éducation des adultes, 8, p. 101-120.

Marcel, J.-F. (Ed.) (2015). La recherche-intervention par les sciences de l'éducation. Accompagner le changement. Dijon : Educagri Editions.

Marcel, J.-F. (2016). Les Sciences de l'éducation à l'école du changement. In J.-L. Rinaudo \& P. Tavignot (Dir.), Le changement à l'école. Sources, tensions, effets (pp. 215-226). Paris :

L'Harmattan.

Marcel, J.-F. (2017). La recherche-intervention comme acte politique et émancipateur In Marcel, J.-F. \& Broussal D. (Eds.). Emancipation et recherche en éducation, p. 397-422, Paris : Editions du Croquant.

Marcel, J-F. (2019a). « Action /connaissance » en sciences de l'éducation : la recherche pour outrepasser les logiques, in Bedin, V., Franc, S. \& Guy, D. (Dir). Les sciences de l'éducation pour quoi faire? Entre action et connaissance, p. 9-19, Paris : L'Harmattan.

Marcel, J.-F. (2019b). Intervention, participation et évaluation dans la recherche en éducation. La Revue LEeE, 1. 
Marcel, J.-F. (2019c). D'aujourd'hui à demain : une discipline sous l'égide de Janus. Les Sciences de l'éducation - Pour l'ère nouvelle, 52-2, p. 29-46.

Marcel, J.-F. (2020). Un dispositif, un tiers-espace et des médiations. Le tiers-espace socioscientifique dans la recherche-intervention in Sciences de la société (à paraître).

Marcel, J.-F. \& Broussal D. (Eds.) (2017). Emancipation et recherche en éducation. Paris : Editions du Croquant.

Marcel, J.-F. \& Bedin, V. (2018). Contribution à l'élaboration d'un dispositif d'évaluation de la Recherche-Intervention. Phronesis, 7 (1), 79-91. https://doi.org/10.7202/1044256ar

Marcel, J-F, Bordes, V. \& Lescouarch, L. (2019). Chercheur-e - Militant-e. Plaidoyer pour un « tiers construit ». Les Dossiers des sciences de l'éducation, 40, 95-104.

Robert, A. D. \& Marcel J-F. (2019). Des relations entre recherches en éducation et engagements militants : éléments pour un débat. In Marcel, J-F, Bordes, V. \& Lescouarch, L. (Eds.), Recherches en éducation et engagements militants. Vers une tierce approche. p. 25-42, Toulouse : PUM.

Peeters, H. \& Charlier, P. (1999). Contributions à une théorie du dispositif. Hermès, La Revue, 3-25, p. 15-23.

Popper, K. (1991). Sur la théorie de l'esprit objectif. In K. Popper (Ed.), La connaissance objective (pp. 245-293). Paris : Aubier. (Première version 1968)

Ricœur P. (2004), Parcours de la reconnaissance. Trois études, Paris : Stock

Robbes, B. (2020). Recherche-action, recherches collaboratives en éducation : une analyse, Distances et médiations des savoirs [En ligne], 29 | 2020, mis en ligne le 21 mars 2020, consulté le 03 avril 2020. URL : http://journals.openedition.org/dms/4707;DOI: https://doi.org/ $10.4000 /$ dms.4707

Vial, M. (2012). Se repérer dans les modèles de l'évaluation: Méthodes - Dispositifs - Outils. Louvain-laNeuve : De Boeck.

\section{NOTES}

1. Il peut sans doute fournir quelques éléments sinon de réponse (ce n'est pas le but) tout au moins d'échanges à la question posée par Bruno Robbes (2020) à propos de la RI et de nos travaux: "Mais en priorisant les visées académique et transformative, l'auteur ne risque-t-il pas de minorer la dimension «avec»?». Le présent article atteste de notre vigilance à ce propos.

2. La RI repose sur la double hypothèse de 1) la conciliation possible des trois visées et leur mise en lien 2) l'enrichissement réciproque de chacune des visées dans et par leurs interrelations.

3. Deux autres dispositifs, fortement participatifs, présentent les mêmes caractéristiques transversales. Le premier est le dispositif de supervision, mis en place au sein de l'UR, qui constitue une ressource et un appui pour les chercheurs engagés, tout au long de la RI et offre un espace précieux et privilégié pour les délibérations éthiques. Le second est la confrontation des participants à des œuvres (Broussal, 2018), l'instauration d'une dialectique entre le particulier et l'universel, d'une passerelle entre le premier et le troisième monde (Popper, 1991) et leurs contributions à une dynamique d'émancipation. 
4. Rappelons que cette participation se fait "avec" des chercheurs qui, eux, sont formés à conduire et même à diriger des recherches.

5. Gurnade et Marcel (1999) qualifient cette catégorie de « savoirs hybrides ».

6. Dans les démarches quantitatives, la généralisation s'obtient grâce au processus, largement codifié, de l'inférence statistique.

7. «Dans cette philosophie pluraliste, le monde est constitué d'au moins trois sousmondes ontologiquement distincts ; ou, dirais-je, il y a trois mondes : le premier est le monde physique, ou le monde des états physiques ; le second est le monde mental, ou le monde des états mentaux ; et le troisième est le monde des intelligibles, ou des idées au sens objectif ; c'est le monde des objets de pensée possibles : le monde des théories en elles-mêmes et de leurs relations logiques ; des argumentations en elles-mêmes ; et des situations de problèmes en elles-mêmes ». (Popper, 1991, p. 247)

8. «La praxéologie se fait dans l'action et par l'action » (Lhotellier et St-Arnaud, 1994, p. 107).

9. «Une praxis est un processus existentiel. L'agir travaille à produire une œuvre, un résultat, mais aussi à changer les relations sociales et l'expression symbolique de l'ensemble », (Lhotellier et St-Arnaud, 1994, p. 99).

10. « l'acteur comme le premier interprète de son agir » (Lhotellier et St-Arnaud, 1994, p. 101).

11. "La praxéologie est moins une conceptualisation d'une pratique que la création d'un savoir nouveau issu de cette pratique » (Lhotellier et St-Arnaud, 1994, p. 95).

12. Le maintien de cette interaction fondatrice permet d'éviter deux écueils, l'un déséquilibré du côté du savoir (scientisme et nominalisme), l'autre du côté de l'action (activisme et empirisme).

13. Nous retrouvons là les quatre piliers de la RI : le sur, le pour, le avec et le par (Marcel, 2015)

14. La mise en place d'un collectif requiert, nous l'avons écrit par ailleurs, deux dimensions irréductibles: l'existence d'une marge d'autonomie (permettant d'élaborer un projet) et l'engagement de chacun de ses membres (l'appartenance à un collectif ne se décrète pas).

15. Projet explicité dans la commande mais sujet à évolutions, de par les différentes appropriations ou de par les imprévus, évolutions acceptables dans le cadre de la plasticité de la commande

16. Principe que nous retrouvons dans la démarche praxéologique: «Dans une perspective de renouveau de l'interaction toujours difficile entre chercheurs et praticiens, la démarche praxéologique entend mettre fin à la tutelle traditionnelle que le savoir homologué imposait à l'agir. L'acteur s'en retrouve valorisé mais aussi confronté à de nouvelles exigences : développer son autonomie et accroître son degré de conscience à tous les niveaux de l'implication sociale » (Lhotellier et St-Arnaud, 1994, p. 107).

17. Nous n'avons pas développé plus avant dans ce texte cette notion que nous mobilisons ici de manière assez littérale, c'est à dire comme une compatibilité concernant d'abord des «objets » épistémologiques, plus précisément les visées, les fonctions, les principes ou les méthodologies de la recherche (ici de la RI), compatibilité investie, qui-plus-est, par une réflexion voire une argumentation également de nature épistémologique.

18. Nous pouvons rajouter des publications dans des revues professionnelles dont la diffusion peut concerner le niveau de l'organisation ou celui de ses réseaux (locaux, régionaux, voire nationaux).

19. Plutôt que de les suspecter a priori : «N'assiste-t-on pas à une forme de récupération par la recherche académique de pratiques pouvant être initiées par les terrains, où les praticiens étaient davantage considérés comme des sujets agissants? ? (Robbes, 2020). 


\section{RÉSUMÉS}

Cet article interroge le rapprochement des fonctions heuristiques et critiques de la recherche en éducation avec la fonction praxéologique, plus précisément, dans le cas d'une démarche participative. L'examen des conditions de la mobilisation des trois fonctions en contexte de participation permet d'analyser leur «épistémo-compatibilité ». L'argumentaire s'appuie sur le cas de la démarche de recherche-intervention, une démarche basée sur l'articulation de visées heuristiques, critiques et praxéologiques indissociables. Ces trois visées sont ancrées dans le principe premier de la participation.Une analyse en deux étapes est mobilisée pour investir l'épistémo-compatibilité de la participation avec chacune des trois fonctions de la recherche en éducation. La première aborde la participation à partir des trois fonctions tandis qu'à l'inverse la seconde investit les visées à partir des trois cercles qui correspondent aux niveaux de participation à l'œuvre dans la recherche-intervention.

This article examines the connection between the heuristic and critical functions of educational research and the praxeological function, more specifically, in the case of a participatory approach. The examination of the conditions of the mobilization of the three functions in the context of participation allows us to analyze their "epistemo-compatibility". The argument is based on the case of the research-intervention approach, an approach based on the articulation of inseparable heuristic, critical and praxeological aims. These three aims are anchored in the primary principle of participation.A two-step analysis is used to examine the epistemic compatibility of participation with each of the three functions of educational research. The first stage approaches participation from the three functions, while the second stage approaches the aims from the three circles that correspond to the levels of participation at work in intervention research.

\section{INDEX}

Mots-clés : participation, recherche-intervention, visées critiques, visées heuristiques, visées praxéologiques

Keywords : participation, research-intervention, critical aims, heuristic aims, praxeological aims

\section{AUTEUR}

\section{JEAN-FRANÇOIS MARCEL}

Professeur, Université Toulouse Jean-Jaurès, UMR EFTS 